\title{
Efficacy and Safety of rt-PA Intravenous Thrombolysis in Patients with Wake- up Stroke: A Meta-Analysis
}

\author{
Hongfa Liu \\ Gannan Medical University \\ Jianghong Luo \\ Gannan Medical University \\ Fang Zhang \\ Gannan Medical University \\ Ziliang Zhang \\ Gannan Medical University \\ Wei Gu \\ Gannan Medical University \\ Jiankun Hong \\ Gannan Medical University \\ Jianping Chen \\ People's Hospital of Ganzhou city \\ Shanquan Zhong \\ Gannan Medical University \\ Ying Huang ( $\sim$ huangying0202@126.com) \\ Gannan Medical University https://orcid.org/0000-0002-4655-5162
}

\section{Research}

Keywords: Meta-analysis, Wake up stroke, Thrombolysis, Efficacy, Safety

Posted Date: April 7th, 2021

DOI: https://doi.org/10.21203/rs.3.rs-371117/v1

License: (c) (1) This work is licensed under a Creative Commons Attribution 4.0 International License. Read Full License

Version of Record: A version of this preprint was published at Medicine on February 18th, 2022. See the published version at https://doi.org/10.1097/MD.0000000000028914. 


\section{Abstract \\ Background}

Recombinant tissue plasminogen activator (rt-PA) is one of the most effective therapies for patients with acute ischemic stroke. However, wake-up stroke (WUS) is typically excluded from intravenous thrombolytic therapy because the time of symptom onset is unclear. Therefore, we aimed to assess the efficacy and safety of rt-PA intravenous thrombolysis in patients with WUS by meta-analysis.

\section{Methods}

We completed a systematic literature search of PubMed, Embase, the Cochrane Library, and SinoMed and included relevant studies of WUS patients covering rt-PA thrombolysis and nonthrombolysis (published from January 1, 2000, to February 28, 2021, with no language restrictions). Primary outcomes included safety outcomes and functional outcomes. Safety outcomes was measured according to the incidence of symptomatic intracranial hemorrhage (SICH) and mortality within 90 days. Efficacy outcomes was measured based on 90-day modified Rankin Scale (mRS) score. We assessed pooled data using either a random-effects model (when $P<0.10, P^{2}>50 \%$ ) or a fixed-effects model (when $P>0.10, P^{2}<50 \%$ ).

\section{Results}

Nine studies with 913 patients were included in the meta-analysis. All patients had ischemic stroke confirmed by computed tomography or magnetic resonance imaging. The incidence of mRS 0-2 was significantly higher in the rt-PA thrombolysis group compared with the nonthrombolysis group. And rt-PA thrombolytic WUS patients did not differ significantly from nonthrombolytic WUS patients in terms of 90-day mortality. However, the rate of SICH was also significantly higher in the rt-PA thrombolysis group than that in the nonthrombolysis group.

\section{Conclusions}

Patients with WUS who received rt-PA thrombolysis had a significant positive effect within 90 days. In addition, although there is no significant increase in mortality, we need to be aware of the risk of intracranial hemorrhage transformation associated with rt-PA thrombolysis despite no obvious increase in mortality. The safety of rt-PA intravenous thrombolysis should be closely monitored in patients with WUS.

\section{Introduction}

Acute ischemic stroke is the second leading cause of death and the third leading cause of disability in the world ${ }^{[1]}$. Approximately one-fifth of ischemic strokes occur during sleep and are not detected until patients wake up with neurological deficits ${ }^{[2,3]}$. Recombinant tissue plasminogen activator (rt-PA) is currently the unique approved treatment for acute ischemic stroke and is restricted to use within $4.5 \mathrm{~h}$ of the onset of ischemic stroke ${ }^{[4]}$. We typically define the last seen normal time as the time of stroke onset. Therefore, WUS has historically been considered a contraindication for rt-PA intravenous thrombolysis ${ }^{[5,6]}$.

However, an increasing number of clinical and imaging observations have demonstrated that a large number of WUS events occur in the early morning and close to the wake-up time ${ }^{[7,8]}$. WUS has similar imaging and clinical characteristics to strokes with known onset times. Moreover, in recent years, several studies have found rt-PA intravenous thrombolysis to be safe and effective in imaging-mismatched patients with WUS ${ }^{[9,10]}$. Although the benefit of rt-PA intravenous thrombolysis in patients with WUS remains controversial, we conducted a meta-analysis of all relevant studies to evaluate the efficacy and safety of rt-PA intravenous thrombolysis in patients with WUS.

\section{Methods}

\subsection{Search Strategy}

We clearly defined our subject of systematic review in terms of populations, interventions, comparators, outcomes and study designs (PICOS). WUS patients as subjects were divided into two groups: one group who received rt-PA intravenous thrombolysis and the control group who received conventional therapy instead. Outcomes included mortality, occurrence of symptomatic intracerebral hemorrhage (SICH) and modified Rankin Scale (mRS) score within 90 days. PubMed, Embase, The Cochrane Library and SinoMed were searched from January 1, 2000, to February 28, 2021. A combination of the following terms was used: "wake up stroke", "wake-up stroke", "stroke on awakening”, "wake up", "recombinant tissue plasminogen activator", "alteplase”, “rtPA", "rt-PA", and "intravenous thrombolysis", "thrombolysis", "reperfusion" and "thrombolytic therapy". For example, the search strategy of PubMed was (intravenous thrombolysis OR thrombolysis OR reperfusion OR thrombolytic therapy) AND (recombinant tissue plasminogen activator OR alteplase OR rtPA OR rt-PA) AND (wake up stroke OR wake-up stroke OR stroke on awakening OR wake up). We also conducted a manual search. We then screened the studies based on title, abstract, and full-text reading. The search strategy was developed without any language restrictions. Ethical approval and patient consent were not required given that the study was based on published articles.

\subsection{Inclusion and Exclusion Criteria}

According to the PICOS principle, all studies were listed according to the following criteria: (1) WUS patients with no restrictions on sex, age, race, or nationality, (2) subjects of the studies included WUS patients who received rt-PA intravenous thrombolysis (total of $0.9 \mathrm{mg} / \mathrm{kg}$ rt-PA), (3) matched controls were defined as 
WUS patients who were not treated with rt-PA intravenous thrombolysis or received standard treatment, (4) the efficacy of rt-PA intravenous thrombolysis was assessed with a 90-day mRS score. Safety was measured by mortality and SICH within 90 days after rt-PA intravenous thrombolysis, and (5) studies had intact data and were published. The exclusion criteria were as follows: (1) conference proceedings, correspondence, case reports, reviews, or preclinical studies, (2) studies lacking critical key information, such as no control group, loss of therapeutic details and basic characteristics of patients, (3) studies with repeated report analysis, and (4) studies missing important data.

\subsection{Quality Assessment}

The quality assessment of nonrandomized studies was executed using the methodological index for nonrandomized studies (MINORS) ${ }^{[11]}$, which was recommended by the Cochrane Working Group on Nonrandomized Research Methods. There were 12 items, and each item was assigned a score of $0-2$. A score of 0 indicates that the study was not reported. A score of 1 indicates that the study was reported but the information was insufficient. A score of 2 indicates that the study was reported and sufficient information was provided. Two researchers (H. F. Liu and Y. Huang) performed quality assessments independently, and disagreements were resolved by consensus.

\subsection{Data Extraction}

Data were extracted by two authors independently. Any divergence was resolved by discussion between two authors and determined eventually by senior authors. Data on authors, publication year, trial design, study period, intervention and comparisons, participant features (number, age, sex, disease history, Toast classification, neuroimaging methods, and NIHSS scores), comorbidities (transient ischemic attack, atrial fibrillation, coronary heart disease, hypertension, and diabetes mellitus), NIHSS scores, admission time and clinical endpoints, including mRS, mortality and the rate of SICH, were collected.

\subsection{Statistical analysis}

All results are presented as odds ratios (ORs) and 95\% confidence intervals (Cls). The heterogeneity of the studies was evaluated by the chi-squared test and qualified by $P$ statistics. When $P<50 \%$ and $P>0.10$, the fixed effects model was used to analyze the results, which demonstrated low heterogeneity among studies. Otherwise, a random effects model was applied in the analysis. Two-sided $P$-values of 0.05 were considered statistically significant. Publication bias was assessed by funnel plots. Sensitivity analyses were conducted to elucidate the effect of each individual study on the overall estimate by removing each study in turn. All statistical analyses were performed using Review Manager (RevMan, version 5.3.5).

\section{Results}

\subsection{Study selection}

The flowchart of the systematic literature search is presented in Figure 1. We identified potentially relevant studies, including 88 from PubMed, 85 from Embase, 63 from Cochrane, and 44 from SinoMed. A total of 271 studies were excluded after reading the title, abstract and full-text using the formulated inclusion and exclusion criteria. Finally, a total of 9 studies ${ }^{[10,12-19]}$ with 913 patients, including 376 patients $(376 / 913,41.18 \%)$ treated with rt-PA thrombolysis, were included in the meta-analysis. Nine studies were all retrospective studies. The baseline characteristics of the WUS patients included in these studies are summarized in Table 1. No significant differences between the WUS thrombolysis group and the WUS nonthrombolysis group were noted in terms of age, sex, previous history or risk factors of stroke patients. The MINORS score results of the quality evaluation are simultaneously presented in Table 1.

\subsection{Outcome assessment}

All results are shown in Figure 2. WUS patients who received rt-PA intravenous thrombolysis presented a higher rate of 90-day mRS 0-2 than nonthrombolysis WUS patients (43.35\% vs $36.13 \%)$. The overall analysis of 90 -day mRS $0-2$ outcome showed a pooled OR of $1.74(95 \% \mathrm{Cl} 1.30-2.34, P=0.0002)$. For safety outcomes, eight studies including 848 patients reported 90-day mortality. We did not identify a significant difference in 90 -day mortality (9.83\% vs $6.97 \%$ ) with a pooled OR of $1.53(95 \% \mathrm{Cl} 0.63-3.76, P=0.35)$. However, the rate of $\mathrm{SICH}$ was significantly increased in the rt-PA intravenous thrombolysis group compared with the group that did not receive rt-PA intravenous thrombolysis $(2.93 \%$ vs $0.56 \%)$ with a pooled OR of $3.03(95 \% \mathrm{Cl} 1.07-8.58, P=0.04)$.

\subsection{Heterogeneity, Sensitivity Analysis, and Publication Bias Assessment}

No obvious heterogeneity among the results of 90-day mRS 0-2, 90-day mRS 0-1 and the incidence of SICH. However, relatively high heterogeneity was noted within 90-day mortality $(P=48 \% ; P=0.06)$. Therefore, we used the leave-one-out method to make sensibility analysis. We found no significant changes in primary outcomes of 90-day mortality, the rate of $\mathrm{SICH}$, the proportion of mRS 0-2 after each study was extracted one by one. In these included studies, we did not identify significant publication bias because the funnel plots were symmetrically distributed (Figure 3).

\section{Discussion}

Based on previous experience, patients with an unknown time of stroke onset, including WUS, were ineligible for thrombolysis treatment according to routine clinical practice guidelines ${ }^{[20]}$. Nevertheless, in recent years, traditional concepts have been gradually shattered based on the generation and development of new diagnostic neuroimaging techniques ${ }^{[21]}$. The time window for evaluating the presence of ischemic penumbra in tissue has gradually taken the place of the traditional time window. This viewpoint has implications for intravenous thrombolysis for ischemic stroke of unknown duration, including WUS patients. As demonstrated in the EXTEND trial ${ }^{[22]}$, patients with ischemic stroke who received intravenous thrombolysis between 4.5 and $9.0 \mathrm{~h}$ after stroke onset or when they awoke from stroke onset symptoms had improved functional outcomes at 90 days. Similar findings were confirmed in the WAKE-UP trial[23], in which patients who received intravenous thrombolysis had favorable 90-day functional outcomes compared with placebo. These patients had an unknown 
time of stroke onset, a magnetic resonance imaging (MRI) mismatch, and MRI findings of an ischemic focus on diffusion-weighted imaging with no significant visible signal changes in the corresponding region on fluid attenuation inversion recovery imaging. In addition, the 2019 American Stroke Association guideline recommended that rt-PA intravenous thrombolysis could benefit WUS patients when the onset of deficit symptoms is close to awakening or when there is a brain imaging mismatch and given Ilb recommendations ${ }^{[24]}$. Our meta-analysis also demonstrated that WUS patients treated with rt-PA intravenous thrombolysis had a higher rate of the 90-day good outcome (mRS 0-2) without increased mortality than nonthrombolysis WUS patients. Thus, we can consider that rt-PA intravenous thrombolysis in WUS patients is moderately effective. However, it is still regretful that the WAKE-UP and EXTEND trials were not included in our meta-analysis because not all patients in these two trials were WUS patients, which did not meet our inclusion criteria.

To our knowledge, similar articles have been published in the last two years. The difference is that their research subjects were stroke patients with unknown onset times rather than WUS ${ }^{[25,26]}$. In addition, rt-PA intravenous thrombolysis increased the rate of SICH in WUS patients in our final outcome assessment, which was inconsistent with previous similar research findings. However, we do not think that rt-PA intravenous thrombolysis treatment is unreliable for WUS patients. There are several possible reasons for the different results. On the one hand, standard imaging inclusion criteria were not employed among the nine included studies, and the imaging criteria included in the meta-analysis varied from noncontrast CT alone to computed tomography perfusion imaging or brain MRI. Previous studies did not follow the imaging criteria for "imaging mismatch", resulting in many WUS patients not receiving timely rt-PA intravenous thrombolysis. This difference may lead to heterogeneity and bias in the final results. On the other hand, retrospective studies have limitations, such as the fact that most of the included studies did not specify the number of losses to follow-up or the rate of loss to follow-up, which may have interfered with our analysis of the results. Based on the above discussion, our clinicians should inform WUS patients and their relatives of the risk of SICH before thrombolysis.

Further limitations should be noted. First, most of the studies we included were nonrandomized because there is a lack of randomized controlled trials in WUS patients domestically and abroad. Second, the sample size of the included studies was small, and the samples were from different regions, which influenced the determination of forest plots and funnel plots for some indicators. Third, the time window of rt-PA intravenous thrombolysis in WUS patients was not uniform in the included studies. Finally, the pre-examination imaging methods were not consistent across all the included studies.

\section{Conclusion}

WUS patients who received rt-PA intravenous thrombolysis had significantly better outcomes at 90 days than those who did not receive thrombolysis, and with no significant increase in mortality. Moreover, it is undeniable that the possibility of rt-PA intravenous thrombolysis treatment should be actively considered for WUS patients as early as possible and monitor closely hemorrhagic transformation. Finally, due to limitations of retrospective studies and the potential heterogeneity among the included studies, the results of current analysis should be further confirmed by new and larger randomized controlled trials in the future.

\section{Declarations}

Competing interests[The authors have no competing interests to disclose.

Funding $\square$ The study was supported by the Open Project of Key Laboratory of Prevention and Treatment of Cardiovascular and Cerebrovascular Diseases, Ministry of Education (XN201816), the Jiangxi Provincial Natural Science Foundation (No. 20202BABL206046), the Gannan District Clinical Research Innovation Team for Geriatric Diseases (\#TD201707 and \#TD201905), and the Key Project of Gannan Medical University (ZD201833).

\section{Authors' contributions[}

Ying Huang: Funding collection, Study design. Hongfa Liu: Writing original draft, Software. Shanquan Zhong: Supervision, Data curation. Jianping Chen: Writing review and editing, Supervision. Ziliang zhang, Fang Zhang, Wei Gu and Jiankun Hong: Data Collection, Literature Search. Jianghong Luo: Statistical Analysis, Data Interpretation.

Ethics approval and consent to participate】All analyses were based on previous published studies, thus no ethical approval and patient consent are required.

Acknowledgments $\unrhd$ The authors thank AJE for edits and revision of this manuscript.

Consent for publication $\square$ Not applicable.Availability of data and materials $\square$ Not applicable.

\section{References}

[1]Lozano R, Naghavi M, Foreman K, et al. Global and regional mortality from 235

causes of death for 20 age groups in 1990 and 2010: a systematic analysis for the

Global Burden of Disease Study 2010. Lancet 2012; 380(9859): 2095-2128.

[2]Elfil M, Eldokmak M, Baratloo A, et al. Pathophysiologic mechanisms, neuroimaging and treatment in wake-up stroke. CNS Spectr 2020; 25(4): 460-467.

[3]Mackey J, Kleindorfer D, Sucharew H, et al. Population based study of wake-up 
strokes. Neurology 2011;76: 1662-1667.

[4]Hacke W, Kaste M, Bluhmki E, et al. Thrombolysis with alteplase 3 to 4.5 hours after acute ischemic stroke. The New England Journal of Medicine 2008; 359(13): 1317-1329.

[5]Powers WJ, Rabinstein AA, Ackerson T, et al. 2018 Guidelines for the Early Management of Patients With Acute Ischemic Stroke: A Guideline for Healthcare Professionals From the American Heart Association/American Stroke Association. Stroke 2018; 49(3): 46-110.

[6]Moradiya Y, Janjua N. Presentation and outcomes of "wake-up strokes" in a large randomized stroke trial: analysis of data from the International Stroke Trial. J Stroke Cerebrovasc Dis 2013; 22(8): 286-292.

[7]Pandya A囚Eggman AA囚Kamel H囚et al. Modeling the Cost Effectiveness of Neuroimaging-Based Treatment of Acute Wake-Up Stroke. PLoS One 2016; 11(2):

e0148106.

[8]Biggs D, Silverman ME, Chen F, et al. How should we treat patients who wake up with a stroke? A review of recent advances in management of acute ischemic stroke. Am J Emerg Med 2019; 37(5): 954-959.

[9]Denny MC, Boehme AK, Dorsey AM, et al. Wake-up strokes are similar to known-onset morning strokes in severity and outcome. J Neurol Neurol Disord 2014; 1(1): 102.

[10]Breuer L, Schellinger PD, Huttner HB, et al. Feasibility and safety of magnetic resonance imaging-based thrombolysis in patients with stroke on awakening: initial single-centre experience. Int J Stroke 2010; 5(2): 68-73.

[11]Slim K, Nini E, Forestier D, et al. Methodological index for non-randomized studies (minors): development and validation of a new instrument. ANZ J Surg 2003; 73(9) : 712-716.

[12]Anaissie JE, Monlezun DJ, Siegler JE, et al. Intravenous Tissue Plasminogen Activator for Wake-Up Stroke: A Propensity Score-Matched Analysis. J Stroke Cerebrovasc Dis 2016; 25(11): 2603-2609.

[13]Bal S, Bhatia R, Shobha N, et al, Stroke-on-Awakening: Safety of CT-CTA Based Selection for Reperfusion Therapy. Can J Neurol Sci 2014; 41(2): 182-186. [14]Barreto AD, Martin-Schild S, Hallevi H, et al. Thrombolytic therapy for patients who wake-up with stroke. Stroke 2009; 40(3): 827-832.

[15]Furlanis G, Ajčević M, Stella AB, et al. Wake-up stroke: thrombolysis reduces ischemic lesion volume and neurological deficit. J Neurol 2020; 267(3): 666-673.

[16]Manawadu D, Bodla S, Jarosz J, et al. A case-controlled comparison of 
thrombolysis outcomes between wake-up and known time of onset ischemic stroke

patients. Stroke 2013; 44(8): 2226-2231.

[17]Roveri L, Gioia SL, Ghidinelli C, et al. Wake-up stroke within 3 hours of symptom awareness: imaging and clinical features compared to standard recombinant

tissue plasminogen activator treated stroke. J Stroke Cerebrovasc Dis, 2013; 22(6):

703-708.

[18]Yuqing Li and Peilan Zhang. Safety and efficacy of recombinant tissue plasminogen activator intravenous thrombolysis in patients with wake-up stroke. Journal of Applied Medicine 2016; 32(22): 3765-3768. [In Chinese] doi 『

10.3969/j.issn.1006-5725.2016.22.037.

[19]Jun Liu, Jianying Sun. MRI-guided thrombolytic therapy of intravenous

ateproxenase in patients with post-awake stroke. International Journal of Cerebrovascular Diseases 2018; 26(10): 721-725. [In Chinese] doi:10.3760/cma.j.issn.1673-4165.2018.10.001.

[20]Silva GS, Lima FO, Camargo EC, et al. Wake-up stroke: clinical and neuroimaging characteristics. Cerebrovascular Diseases 2010; 29(4): 336-342.

[21]Cho A, Sohn S, Han M, et al. Safety and Efficacy of MRI-Based Thrombolysis in

Unclear-Onset Stroke. Cerebrovascular Diseases 2008; 25(6): 572-579.

[22] Ma H, Campbell BCV, Parsons MW, et al. Thrombolysis Guided by Perfusion Imaging up to 9 Hours after Onset of Stroke. The New England journal of medicine. 2019; 380(19): 1795-1803.

[23]Thomalla G, Simonsen CZ, Boutitie F, et al. MRI-Guided Thrombolysis for Stroke with Unknown Time of Onset. The New England journal of medicine. 2018; 379(7): 611-622.

[24]Powers WJ, Rabinstein AA, Ackerson T, et al. Guidelines for the Early

Management of Patients With Acute Ischemic Stroke: 2019 Update to the 2018

Guidelines for the Early Management of Acute Ischemic Stroke: A Guideline for

Healthcare Professionals From the American Heart Association/American Stroke

Association. Stroke 2019; 50(12): 344-418.

[25]Luan D, Zhang Y, Yang Q, et al. Efficacy and Safety of Intravenous Thrombolysis in Patients with Unknown Onset Stroke: A Meta-Analysis. Behavioural Neurology 2019; 2019: 5406923.

[26]Zhu RL, Xu J, Xie CJ, et al. Efficacy and Safety of Thrombolytic Therapy for Stroke with Unknown Time of Onset: A Meta-Analysis of Observational Studies. J Stroke Cerebrovasc Dis 2020; 29(5): 104742.

\section{Tables}


Table 1. The baseline characteristics of the included studies

\begin{tabular}{|c|c|c|c|c|c|c|c|c|c|}
\hline Study & Anaissie $2016^{\square 12 \square}$ & Bal2014 & Barreto2009 & Breuer2010 & $\begin{array}{c}\text { Furlanis } 2019 \\
\square 15 \square\end{array}$ & Jun Liu2018 & $\begin{array}{c}\text { Yuqing Li2016 } \\
\square 18 \square\end{array}$ & Manawadu2013 & Roveri \\
\hline$\overline{\text { Design }}$ & Retrospective & Retrospective & Retrospective & Retrospective & Retrospective & Retrospective & Retrospective & Retrospective & $\overline{\text { Retro: }}$ \\
\hline Period & 2008.07-2014.05 & 2003.01-2010.03 & 2003.03-2008.01 & $\begin{array}{l}2006.10- \\
2008.05\end{array}$ & $\begin{array}{l}2013.11- \\
2018.12\end{array}$ & 2016.05-2018.06 & 2015.06-2016.06 & 2009.01-2010.12 & 2005.0? \\
\hline No.of patients & $46 / 154$ & $29 / 41$ & $46 / 34$ & $10 / 35$ & $74 / 75$ & $30 / 35$ & $64 / 58$ & $68 / 54$ & 9 \\
\hline Age (y) & $\begin{array}{l}69(42-98) / \\
63(22-93) \mathrm{b}\end{array}$ & $68(23) / 74(21)^{c}$ & $62 \pm 14 / 64 \pm 13^{a}$ & $\begin{array}{l}73(57-92) / \\
66(39-87)^{b}\end{array}$ & $73 \pm 13 / 79 \pm 10^{a}$ & $\begin{array}{c}66.0 \pm 9.3 / \\
65.8+108^{a}\end{array}$ & $\begin{array}{c}62.9 \pm 2.1 / \\
65.9+113^{a}\end{array}$ & Not reported & $\begin{array}{l}59.221 \\
70.431\end{array}$ \\
\hline Gender(male,\%) & $22(47.9) / 85(55.2)$ & $12(41) / 17(41)$ & $11(39) / 15(44)$ & $5(50) / 23(66)$ & $30(41) / 39(52)$ & $18(60.0 / 20(57.1)$ & $38(59.4) / 33(56.9)$ & $23(33.8) / 28(51.9)$ & $33(55)$ \\
\hline $\begin{array}{l}\text { Admission NIHSS } \\
\text { score }\end{array}$ & $9.5(1-27) / 5(0-33)^{b}$ & $14(11) / 13(11)^{\mathrm{c}}$ & $\begin{array}{c}16(3-24) / \\
10.5(2-26)^{\mathrm{b}}\end{array}$ & $\begin{array}{c}10.5(1-22) / 6(1- \\
21)^{\mathrm{b}}\end{array}$ & $\begin{array}{c}6(4-13) / 7(3- \\
16)^{b}\end{array}$ & Not reported & $8.7 \pm 5.2 / 9.2 \pm 4.9^{a}$ & $11(8-17) / 9.5(5-16)^{b}$ & $9(4-19)$ \\
\hline Hypertension $\square \mathrm{n} \square \% \square$ & $36(78.3) / 119(77.3)$ & $18(62.1) / 27(65.9)$ & $30(65) / 22(65)$ & $9(90) / 29(83)$ & $53(72) / 66(88)$ & $19(63.3) / 23(65.7)$ & $25(39.1) / 23(39.7)$ & $45(66.2) / 32(59.3)$ & $36(60)$ \\
\hline $\begin{array}{l}\text { Diabetes mellitus } \\
\square \mathrm{n} \square \% \square\end{array}$ & $20(43.5) / 45(29.2)$ & $3(10.3) / 7(17.1)$ & $10(21) / 11(33)$ & $4(40) / 13(37)$ & $15(20) / 26(35)$ & $8(26.7) / 11(31.4)$ & $20(31.3) / 19(32.8)$ & $14(20.6) / 13(24.1)$ & $14(23.3)$ \\
\hline $\begin{array}{l}\text { Cardiovascular } \\
\text { disease } \llbracket n \rrbracket \% \square\end{array}$ & Not reported & Not reported & $8(17) / 5(15)$ & $3(30) / 6(17)$ & $15(20) / 22(29)$ & $10(33.3) / 13(37.1)$ & $23(35.9) / 21(36.2)$ & Not reported & $24(40)$ \\
\hline Smoking $\square \mathrm{n} \square \% \square$ & Not reported & $13(44.8) / 19(46.3)$ & Not reported & Not reported & $15(20) / 21(25)$ & $10(33.3) / 14(40.0)$ & $29(45.3) / 25(43.1)$ & Not reported & $12(20.7)$ \\
\hline $\begin{array}{l}\text { Previous } \\
\text { stroke/TIA } \square n \square \% \square\end{array}$ & Not reported & Not reported & Not reported & $5(50) / 9(26)$ & Not reported & $3(10.0) / 5(14.3)$ & Not reported & Not reported & $14(23.3)$ \\
\hline Dyslipidemia $\square \mathrm{n} \square \% \square$ & $19(41.3) / 60(39)$ & $9(31.0) / 6(14.6)$ & $12(27) / 9(27)$ & $8(80) / 27(77)$ & $47(64) / 47(63)$ & $18(60.0) / 20(57.1)$ & $18(28.1) / 15(25.9)$ & $22(32.4) / 25(46.3)$ & $19(31.7)$ \\
\hline Imaging criteria & $\begin{array}{l}\text { Early ischemic } \\
\text { changes of } 1 / 3 \\
\text { MCA territory }\end{array}$ & $\underset{\text { CT }}{\text { ASPECTS }}>7$ on & $\begin{array}{l}\text { Early ischemic } \\
\text { changes of } 1 / 3 \\
\text { MCA territory }\end{array}$ & $\begin{array}{l}\text { PWI/DWI } \\
\text { mismatch:DWI } \\
\text { volume } 1 / 3 \\
\text { MCA territory }\end{array}$ & $\begin{array}{c}\text { ASPECTS > } 6 \\
\text { on CT and/or } \\
\text { ischemic } \\
\text { penumbra }> \\
50 \% \text { of } \\
\text { hypoperfused } \\
\text { tissue on CTP }\end{array}$ & $\begin{array}{l}\text { DWI/FLAIR } \\
\text { mismatch }\end{array}$ & $\begin{array}{l}\text { DWI/FLAIR } \\
\text { mismatch }\end{array}$ & $\begin{array}{l}\text { Early ischemic } \\
\text { changes of } 1 / 3 \\
\text { MCA territory }\end{array}$ & $\begin{array}{l}\text { Early } \mathrm{i} \\
\text { change } \\
\text { MCA t }\end{array}$ \\
\hline Admission time(h) & 4.5 & Not reported & 3 & 6 & 4 & 4.5 & 4.5 & 4.5 & \\
\hline $\begin{array}{l}\text { MINORS scale } \\
\text { score }\end{array}$ & 20 & 21 & 20 & 22 & 21 & 22 & 22 & 24 & \\
\hline
\end{tabular}

a: mean $\pm \mathrm{SD}$, b: median (minimum- maximum), c: mean ( interquartile)

\section{Figures}

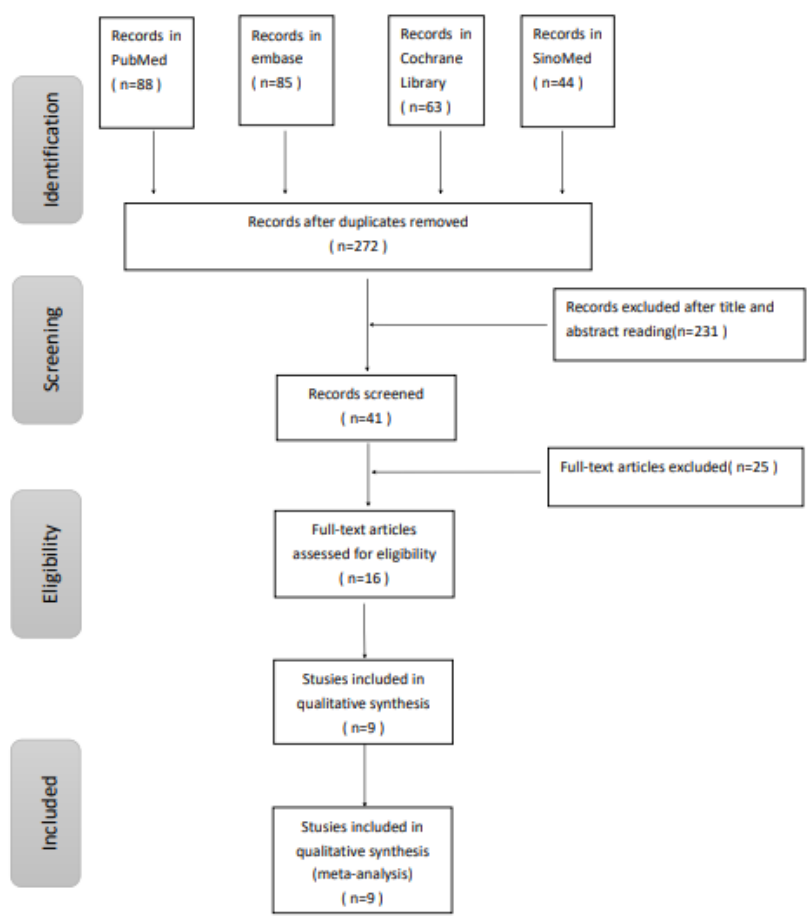

\section{Figure 1}

Flowchart of the study selection. 

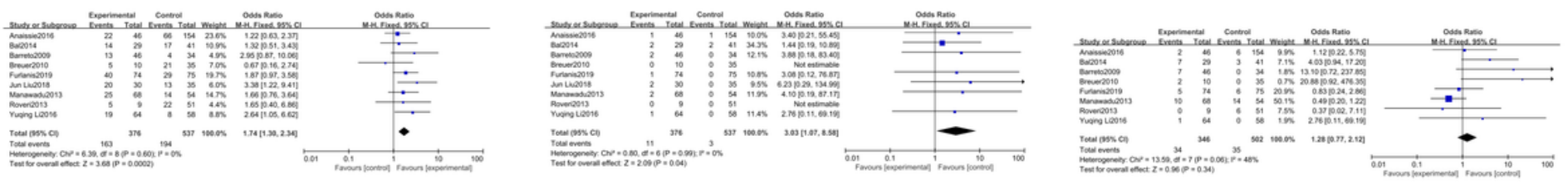

\section{Figure 2}

(a). Forest plot of the rate of mRS(0-2) within 90 days between experimental group and control group. (b). Forest plot of the rate of SICH within 90 days between experimental group and control group. (c). Forest plot of the mortality within 90 days between experimental group and control group.
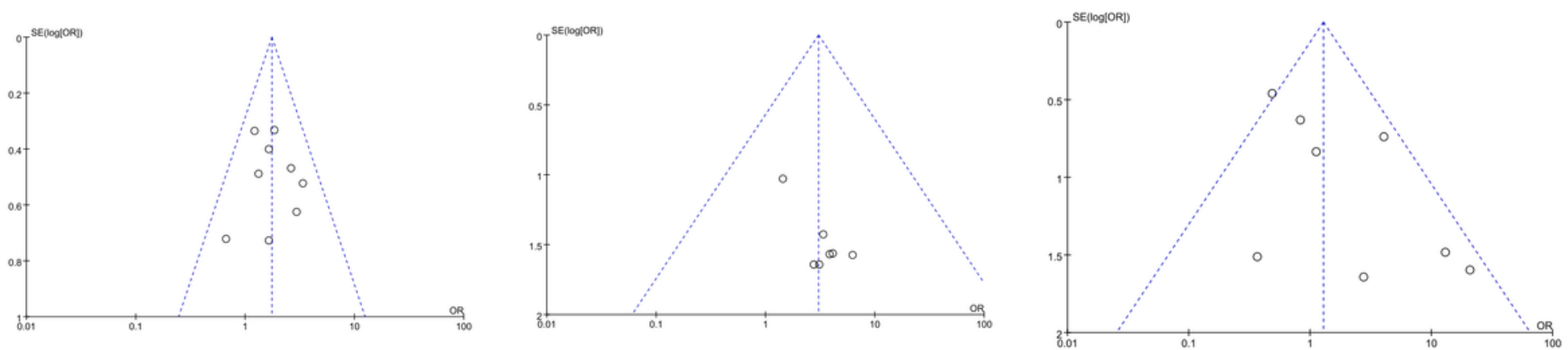

\section{Figure 3}

(a). Funnel plot of the rate of $\mathrm{mRS}(0-2)$ within 90 days between experimental group and control group. (b). Funnel plot of the rate of SICH within 90 days between experimental group and control group. (c). Funnel plot of the mortality within 90 days between experimental group and control group. 